\title{
Bilateral ovarian carcinomas differ in the expression of metastasis-related genes
}

\author{
MARIANNE LISLERUD SMEBYE ${ }^{1,2}$, LISBETH HAUGOM $^{1,2}$, BEN DAVIDSON $^{3,4}$, CLAES GÖRAN TROPE $^{5}$, \\ SVERRE HEIM $^{1,2,4}$, ROLF INGE SKOTHEIM ${ }^{2,6}$ and FRANCESCA MICCI ${ }^{1,2}$

\begin{abstract}
${ }^{1}$ Section for Cancer Cytogenetics, Institute for Cancer Genetics and Informatics, The Norwegian Radium Hospital, Oslo University Hospital, NO-0424 Oslo; ${ }^{2}$ Centre for Cancer Biomedicine, Faculty of Medicine, University of Oslo, NO-0316 Oslo; ${ }^{3}$ Department of Pathology, The Norwegian Radium Hospital, Oslo University Hospital, NO-0424 Oslo;

${ }^{4}$ Institute of Clinical Medicine, Faculty of Medicine, University of Oslo, NO-0316 Oslo; ${ }^{5}$ Department of Gynecology, The Norwegian Radium Hospital, Oslo University Hospital; ${ }^{6}$ Department of Molecular Oncology,

Institute for Cancer Research, The Norwegian Radium Hospital, Oslo University Hospital, NO-0424 Oslo, Norway
\end{abstract}

Received November 5, 2015; Accepted July 1, 2016

DOI: $10.3892 / \mathrm{ol} .2016 .5384$

\begin{abstract}
The mechanisms behind bilaterality of ovarian carcinomas are not fully understood, as the two tumors could possibly represent two primary tumors, a primary tumor and a metastasis, or two metastases. The gene expression profiles from bilateral high-grade serous carcinomas (HGSCs) and clear cell carcinomas (CCCs) of the ovary were compared to study the association between the tumors of the two sides. A separate analysis of genes from chromosome 19 was also performed, since this chromosome is frequently rearranged in ovarian carcinomas. Tumors from four patients were included (three pairs of HGSC and one pair of CCC). The gene expression was analyzed at the exon level, and bilateral tumors were compared to identify within-pair differences. Gene expression data were also compared with genomic information on the same tumors. Similarities in gene expression were observed between the tumors within each pair, as expected if the two tumors were clonally related. However, certain genes exhibited differences in expression between the two sides, indicating metastasis involvement. Among the most differently expressed genes, one gene was common to all four pairs: Immunoglobulin J. In all HGSC pairs, serpin peptidase inhibitor, clade B (ovalbumin), member 2, serpin family E member 1 and phospholipase A2, group IIA (platelets, synovial fluid) were also among the differentially expressed genes. The specific analysis
\end{abstract}

Correspondence to: Dr Francesca Micci, Section for Cancer Cytogenetics, Institute for Cancer Genetics and Informatics, The Norwegian Radium Hospital, Oslo University Hospital, Ullernchausseen 70, P. O. Box 4953 Nydalen, NO-0424 Oslo, Norway

E-mail: francesca.micci@medisin.uio.no

Key words: ovarian carcinoma, metastasis, bilateral, gene expression of chromosome 19 highlighted expression differences in the zinc finger protein 36 gene. These results indicate that bilateral ovarian tumors represent different stages during progression of a single clonal process. Several of the genes observed to be differently expressed are known to be metastasis-related, and are likely to be also involved in spreading from one side to the other in the bilateral cancer cases examined.

\section{Introduction}

Ovarian cancer is the seventh most common cancer in women, and affects 239,000 women worldwide each year (1); however, the natural history of these cancers as well as their underlying tumor biology are poorly understood (2). Carcinomas are the most frequent type of ovarian cancer, which can be divided into five main types: High-grade serous carcinoma (HGSC; accounting for $70 \%$ ), clear cell carcinoma (CCC; 10\%), endometrioid carcinoma (10\%), mucinous carcinoma (3\%) and low-grade serous carcinoma $(<5 \%)$ (3). At the time of surgery, cancer involves both ovaries in $66 \%$ of patients with HGSC and in $8 \%$ of patients with CCC (4). The cell type(s) of origin in ovarian carcinogenesis is(are) unknown. It is debated whether all HGSC arise in the fallopian tubes or if a number of them stem from the surface epithelium of the ovaries $(2,5)$. In CCC, possible origins are the ovarian surface epithelium or cells from the endometrium, as the latter tumor type is associated with endometriosis in $\sim 1 / 3$ of the patients (4). We hypothesize that the developmental history of bilateral ovarian carcinomas likewise represents a conundrum; in principle, it may represent two primary tumors, a primary tumor and its metastasis, or two metastases.

Genomic studies of bilateral carcinomas have revealed a clonal association between the two tumors in each patient, including similarities in karyotype and copy number aberrations (6-8). Gene expression analyses of ovarian tumors and their omental metastases have also demonstrated similarities in gene expression profiles among the lesions, with a number of the differently expressed genes being linked to the metastatic 
process (9-11). To the best of our knowledge, no comparison at the transcriptome level to search specifically for genes differently expressed between the two tumors in bilateral ovarian carcinomas has been published thus far.

As genes differentially expressed within bilateral pairs may indicate the developmental association between the two tumors, a microarray analysis of four pairs of ovarian carcinomas (three pairs of HGSC and one pair of CCC) was conducted. The results were compared with previous genomic data on the same tumors (7) with the aim of identifying possible mechanisms behind the RNA level differences.

The present tumor samples were originally selected because the karyotypes of one of the samples in each tumor pair displayed structural rearrangements of chromosome 19; the karyotype analyses of the four remaining tumors were either inconclusive or culture failure. Chromosome 19 is commonly and non-randomly rearranged in ovarian carcinogenesis (12-15). Since structural rearrangements of chromosome 19 have not been reported as the sole anomaly, these changes are probably linked to tumor progression rather than initiation. Therefore, in addition to the global gene expression data set, a separate analysis of genes mapping on chromosome 19 was performed in the present study.

\section{Materials and methods}

Patients and tissue samples. Bilateral ovarian carcinomas from four patients were included in the present study (three pairs of HGSC and one pair of CCC). Tumor grade and histology were revised by a gynecological pathologist (Table I). The same material had previously been examined by karyotyping and comparative genomic hybridization (CGH) (7). The tumors were surgically removed at The Norwegian Radium Hospital (Oslo, Norway) between January 2001 and December 2004. The samples were originally selected because rearrangement(s) of chromosome 19 had been noticed in the karyotype of one of the bilateral tumors. None of the patients had received chemotherapy prior to surgery. The tumor biobank was registered according to the Norwegian national legislation, and the study was approved by the South East Regional Committee for Medical Research Ethics (Oslo, Norway; project nos. S-07194a and 2.2007.425).

For comparison of technical variation and gene expression levels, one healthy ovary sample (Human Ovary Total RNA; catalogue no. HR-406; Zyagen, San Diego, CA, USA) was analyzed in three separate replicates (N1-3).

Isolation of RNA. The tumor tissue adjacent to that used for cytogenetic analysis and histological examination had been frozen and stored at $-80^{\circ} \mathrm{C}$. Total RNA was extracted using TRIzol reagent (Invitrogen; Thermo Fisher Scientific, Inc., Waltham, MA, USA) and RNAeasy spin columns (RNeasy Mini kit; Qiagen GmbH, Hilden, Germany). First, the tumor tissue was homogenized in TRIzol, and the aqueous phase was then removed and used further with the aforementioned kit, according to the manufacturer's protocol. Quantification and quality control of the isolated total RNA were performed using the NanoVue spectrophotometer (GE Healthcare Life Sciences, Chalfont, UK) and the Experion automated electrophoresis system (Bio-Rad Laboratories, Inc., Hercules, CA,
Table I. Clinical data.

\begin{tabular}{lcccc}
\hline Patient & Lab no. & Histology & FIGO stage & Age $^{\text {a }}$ \\
\hline 1 A/B & $01-805 / 6$ & HGSC & IIIC & 54 \\
$2 \mathrm{~A} / \mathrm{B}$ & $01-837 / 8$ & HGSC & IIIC & 61 \\
$3 \mathrm{~A} / \mathrm{B}$ & $04-186 / 7$ & HGSC & IIC & 64 \\
$4 \mathrm{~A} / \mathrm{B}$ & $04-101 / 2$ & CCC $^{\mathrm{b}}$ & IIIB & 58 \\
\hline
\end{tabular}

${ }^{a}$ Age at time of surgery (years). ${ }^{b}$ Sample previously classified as mixed clear cell/serous histology. Lab, laboratory; HGSC, high-grade serous carcinoma; CCC, clear cell carcinoma; FIGO, International Federation of Gynecology and Obstetrics.

USA). Total RNA degradation was evaluated by reviewing the electropherograms and the RNA quality indicator (RQI) numbers (RQI=7.5-9.0).

Microarray gene expression analysis. Total RNA (100 ng) was used as input for global gene expression analysis at the exon level using GeneChip ${ }^{\circledR}$ Human Exon 1.0 ST Arrays (Affymetrix, Inc., Santa Clara, CA, USA). Each microarray contained 1.4 million probe sets (the majority of which were comprised of four probes), where each probe set corresponded to $\sim 1$ known or computationally predicted exon. RNA from each sample was individually amplified, reversely transcribed, fragmented and labeled. Labeled sense strand complementary DNA was hybridized onto the arrays, which were then washed, stained and scanned as described previously (16). Cell intensity files from the eight tumor samples were background corrected, inter-chip quantile normalized and summarized at the gene level by the robust multiarray average approach (17), implemented in the Expression Console 1.1 software (Affymetrix, Inc.). A total of 17,361 genes (transcript clusters annotated with gene symbols) were identified using the HuEx-1_0-st-v2.r2 core library files and the HuEx-1_0-st-v2.na33.hg19.transcript.csv annotation file (Affymetrix, Inc.). In a second round of normalization, which was performed as described above, the healthy control was normalized together with the tumor samples. The gene expression text file was imported into J-Express Pro 2012 (Molmine, Bergen, Norway) for hierarchical clustering (Euclidean distance measure and complete linkage) (18).

The expression of known ovarian cancer-related genes was specifically evaluated. Genes were selected from the Cancer Gene Census (http://cancer.sanger.ac.uk/census/, accessed on October 12, 2015) by the search term 'ovarian;' and 19 genes were identified, including $A K T 1, A K T 2, A T$-rich interaction domain 1A, BRAF, breast cancer (BRCA)1, BRCA2, cyclin E1, cyclin-dependent kinase 12, catenin beta 1, Erb-B2 receptor yyrosine kinase 2, forkhead box L2, MutL homolog 1, MutS homolog (MSH)2, MSH6, phosphatidylinositol 3-kinase regulatory subunit 1, PMS1, PMS2, protein phosphatase 2, regulatory subunit A, alpha and serine/threonine kinase 11.

\section{Results}

Gene expression. The gene expression analysis provided informative results on 17,361 genes annotated with gene 
Table II. Genes differently expressed within $\geq 2$ pairs of HGSC.

\begin{tabular}{|c|c|c|c|c|c|}
\hline Gene symbol & Ensembl gene ID & Cytoband & $1^{\mathrm{a}}$ & $2^{\mathrm{a}}$ & $3^{\mathrm{a}}$ \\
\hline ACTA2 & ENSG00000107796 & $10 q 23.3$ & 11.09 & 5.83 & 1.04 \\
\hline$B C A T 1$ & ENSG00000060982 & $12 \mathrm{p} 12.1$ & 1.18 & 5.19 & 9.19 \\
\hline$B D K R B 1$ & ENSG00000100739 & $14 q 32.1-q 32.2$ & 10.95 & 4.21 & 1.18 \\
\hline C8orf4 & ENSG00000176907 & $8 \mathrm{p} 11.2$ & 12.16 & 4.39 & 1.66 \\
\hline CRISPLD2 & ENSG00000103196 & $16 q 24.1$ & 5.54 & 5.05 & 1.34 \\
\hline CXCL12 & ENSG00000107562 & $10 q 11.1$ & 9.59 & 5.12 & 1.80 \\
\hline EFEMP1 & ENSG00000115380 & $2 \mathrm{p} 16$ & 11.48 & 8.40 & 2.96 \\
\hline$E I F 2 S 2$ & ENSG00000125977 & $20 q 11.2$ & 3.21 & 4.06 & 4.07 \\
\hline ENPP1 & ENSG00000197594 & $6 q 22-q 23$ & 5.07 & 4.26 & 1.62 \\
\hline EPYC & ENSG00000083782 & $12 q 21$ & 9.07 & 9.75 & 1.09 \\
\hline$F A P$ & ENSG00000078098 & $2 q 23$ & 11.13 & 3.34 & 2.69 \\
\hline$H A S 2$ & ENSG00000170961 & $8 q 24.12$ & 7.54 & 4.58 & 1.17 \\
\hline HISTIHIA & ENSG00000124610 & $6 \mathrm{p} 21.3$ & 5.14 & 1.15 & 6.81 \\
\hline$I G J^{b}$ & ENSG00000132465 & $4 q 21$ & 41.81 & 3.62 & 13.20 \\
\hline$I L 7 R$ & ENSG00000168685 & $5 p 13$ & 5.55 & 3.47 & 1.34 \\
\hline$I N H B A$ & ENSG00000122641 & $7 \mathrm{p} 15-\mathrm{p} 13$ & 4.25 & 3.28 & 1.74 \\
\hline$P L A 2 G 2 A$ & ENSG00000188257 & $1 \mathrm{p} 35$ & 5.48 & 1.45 & 4.07 \\
\hline PTGIS & ENSG00000124212 & $20 q 13.13$ & 6.86 & 5.73 & 1.93 \\
\hline S100A8 & ENSG00000143546 & $1 q 21$ & 7.16 & 1.77 & 7.09 \\
\hline S100A9 & ENSG00000163220 & $1 q 21$ & 4.63 & 1.11 & 5.92 \\
\hline SERPINB2 ${ }^{b}$ & ENSG00000197632 & $18 q 21.3$ & 8.37 & 5.93 & 5.57 \\
\hline SERPINE1 & ENSG00000106366 & $7 q 21.3-q 22$ & 7.35 & 8.38 & 1.99 \\
\hline
\end{tabular}

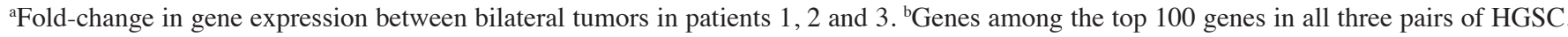
HGSC, high-grade serous carcinomas; ID, identity; ACTA2, actin, alpha 2, smooth muscle, aorta; BCAT1, branched chain amino acid transaminase 1; BDKRB1, bradykinin receptor B1; C8orf4, chromosome 8 open reading frame 4; CRISPLD2, cysteine-rich secretory protein LCCL domain containing 2; CXCL12, C-X-C motif chemokine 12; EFEMP1, EGF containing fibulin-like extracellular matrix protein 1; EIF2S2, eukaryotic translation initiation factor 2, subunit 2 (beta); ENPP1, ectonucleotide pyrophosphatase/phosphodiesterase 1; EPYC, epiphycan; $F A P$, familial adenomatous polyposis; HAS2, hyaluronan synthase 2; HIST1H1A, histone cluster 1, H1a; IGJ, immunoglobulin J; IL7R, interleukin-7 receptor; INHBA, inhibin, beta A (activin A, activin AB alpha polypeptide); PLA2G2A, phospholipase A2, group IIA (platelets, synovial fluid); PTGIS, prostaglandin I2 (prostacyclin) synthase; S100A8, S100 calcium binding protein A8; S100A9, S100 alcium binding protein A9; SERPINB2, serpin peptidase inhibitor, clade B (ovalbumin), member 2; SERPINE1, serpin peptidase inhibitor, clade E, member 1.

A

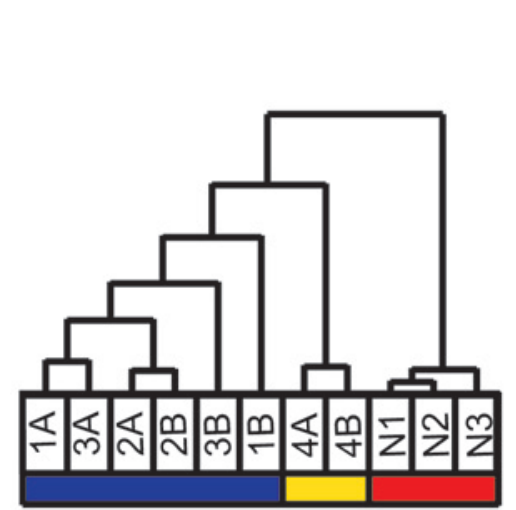

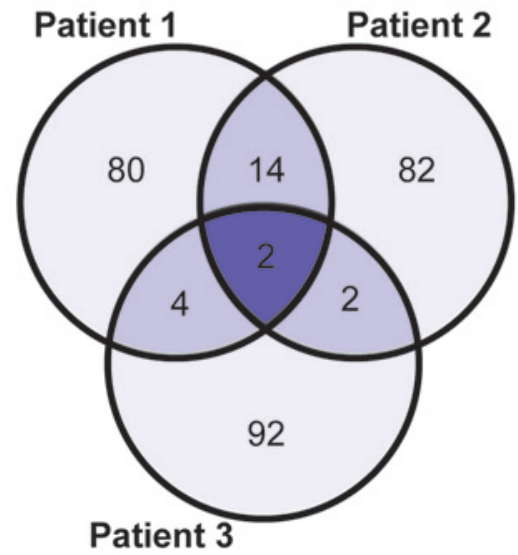

C

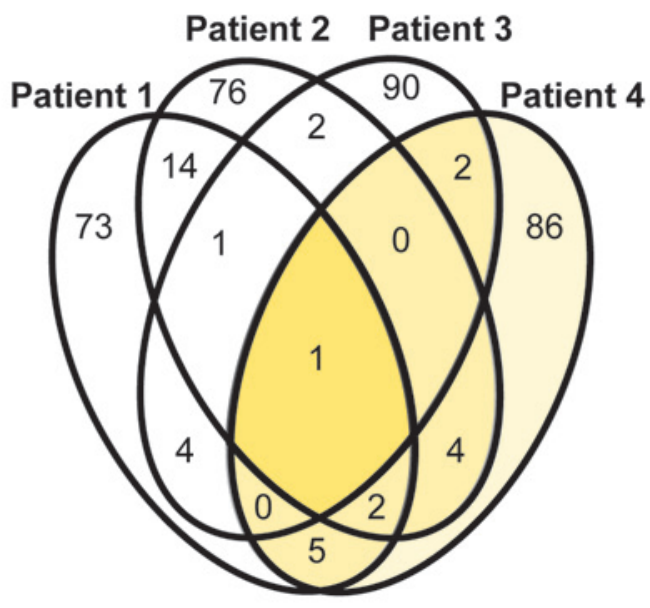

Figure 1. Comparison of samples. (A) Hierarchical clustering of six HGSC (blue), two CCC (yellow) and three normal ovary replicates (red) separated normal tissue from cancer and HGSC from CCC. The clustering is based on the normalized expression levels of the 1,000 genes with the greatest range in expression across the sample set. (B) Comparison of the most differently expressed genes within three pairs of HGSC revealed that 22 genes were differently expressed in $\geq 2$ pairs, two of which were common to all: IGJ and serpin peptidase inhibitor, clade B (ovalbumin), member 2. (C) Comparison of the most differently expressed genes within three pairs of HGSC and one pair of CCC (yellow). One gene was among the most differently expressed in all four pairs: IGJ. HGSC, high-grade serous carcinoma; CCC, clear cell carcinoma; IGJ, immunoglobulin J. 
symbols. The hierarchical clustering of all samples (six HGSC from three patients, two CCC from one patient and three replicates of a healthy whole ovarian tissue) based on all genes separated healthy tissue from cancer, with the CCC samples clustering with the HGSC samples. All bilateral tumors exhibited within-pair clustering. When the hierarchical clustering analysis was based on the 1,000 genes with the greatest range in gene expression, cancer samples were separated from healthy controls and HGSC were separated from CCC (Fig. 1A). Since the aim of the study was to identify genes with different expression within pairs, no further studies on the similarities within tumor pairs were performed.

Comparison of bilateral HGSC. The proportion of genes differently expressed within the three pairs ( $\geq 2$-fold-change between normalized expression values) was 4.0,2.6 and 4.8\%, respectively. For each pair, the top 100 genes differing the most were selected for further analysis. The comparison of these lists identified 276 unique genes (Fig. 1B), including 22 genes among the most differently expressed genes in $\geq 2$ patients (Table II). Two genes were among the most differently expressed within all three pairs: Immunoglobulin J (IGJ, Fig. 2A) and serpin peptidase inhibitor, clade B (ovalbumin), member 2 (SERPINB2, Fig. 2B). Additionally, other genes displayed differences in expression within all pairs, including serpin peptidase inhibitor, clade E, member 1 (SERPINE1, Fig. 2C).

Comparison with CCC and reference tissue. Since the cell(s) of origin in ovarian carcinomas is(are) uncertain, it is important to select the reference tissue carefully. The different histotypes were analyzed separately, and the results from normal whole ovary were used mainly as a control of technical variation. Within the pair of CCC, $1.9 \%$ of the genes differed in expression ( $\geq 2$-fold-change) between the two sides. Of the 100 most differently expressed genes within this pair, one gene was also among the most differently expressed in all three HGSC pairs: $I G J$ (Fig. 1C). In addition to $I G J, 13$ other genes were differently expressed within $\geq 1$ pair of HGSC [tenascin $C$, interleukin (IL)-7 receptor, uroplakin $1 B$, chemokine $(C-C$ motif) ligand 2, small proline-rich protein $2 A$, eyes absent homolog 4, cytochrome P450, family 24, subfamily A, polypeptide 1, insulin-like growth factor 2, lymphocyte antigen 96 , lumican, paired related homeobox 1 and chromosome 8 open reading frame 4]. RNA from normal whole ovary was analyzed in three separate replicates, and only $0.9 \%$ of the total gene set differed $>2$-fold when comparing the replicates.

Comparison with genomic data. The present gene expression results were compared with previously reported genomic analyses of the same material (7). CGH analysis provided copy number information at a resolution level of $\sim 300$ cytobands. All HGSC tumors exhibited identical status in 39 cytobands, specifically, all six had either copy number gain, copy number loss or were balanced/had no alteration. In total, $\geq 1$ tumor sample(s) differed in copy number from the rest in the remaining cytobands. In eight of them (1p35, 1p32, 4p16, 7q11, 11p11, 12q14, 19p13 and 20q11), all three HGSC pairs displayed different results within pairs. In three of these, the same combination was detected in all pairs: 1p35 (loss/no alteration), 11p11 (loss/no alteration) and 20q11 (gain/no alteration).
A

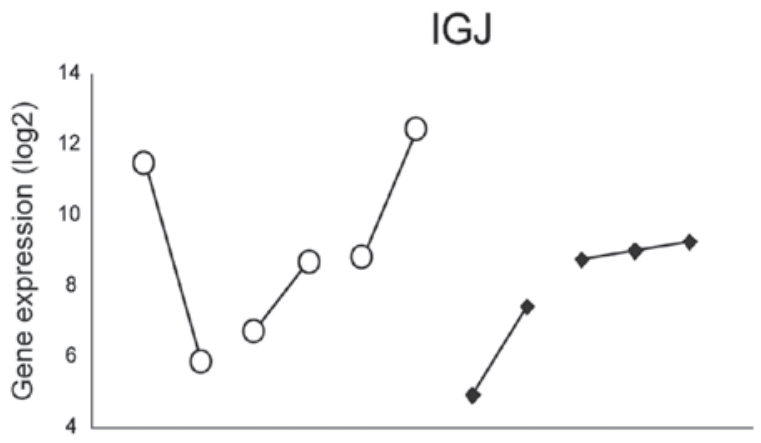

B

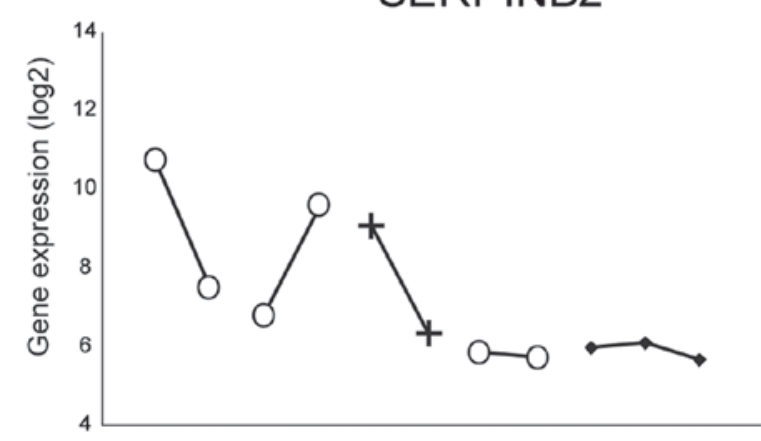

C

SERPINE1

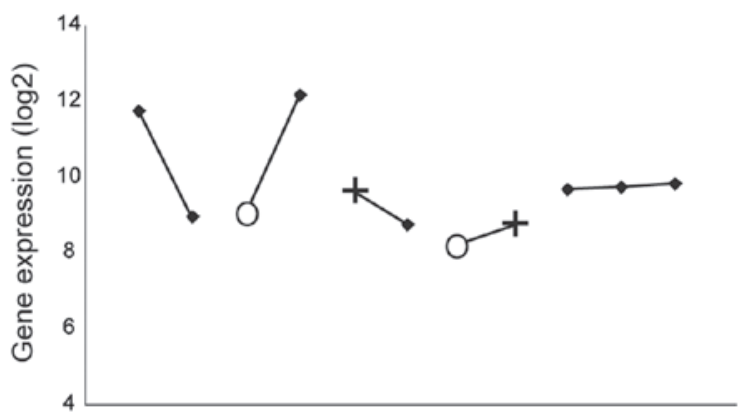

D

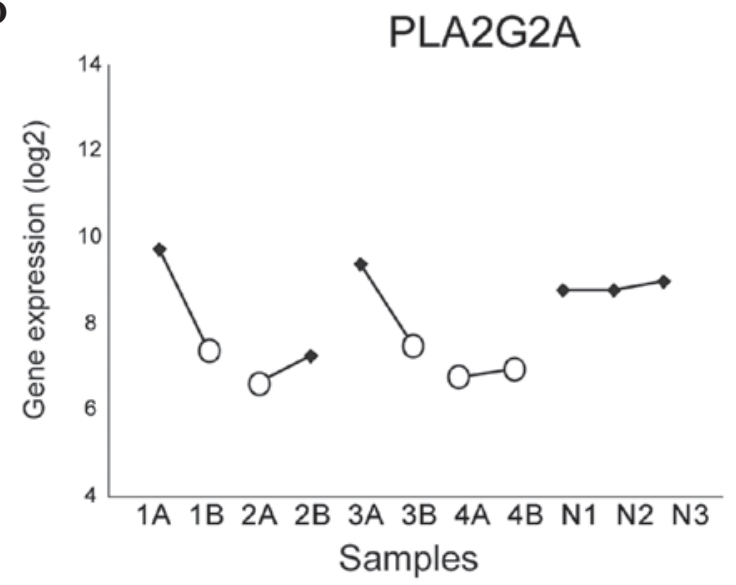

Figure 2. Gene expression in three pairs of HGSC (1-3), one pair of clear cell carcinoma (4) and normal ovarian tissue (replicates N1-3). Comparative genomic hybridization copy number results are marked with $\mathrm{O}$ (loss) and + (gain). (A) Immunoglobulin $J$ exhibited $>4$-fold-change in expression within all tumor pairs. (B) Serpin peptidase inhibitor, clade B (ovalbumin), member 2 was differently expressed in all HGSC pairs, (C) similarly to another member of the same gene family: Serpin peptidase inhibitor clade E, member 1. (D) For phospholipase A2, group IIA (platelets, synovial fluid), an association appeared to exist between DNA copy number loss and reduced gene expression. HGSC, high-grade serous carcinoma; IGJ, immunoglobulin J; SERPINB2, serpin peptidase inhibitor, clade B (ovalbumin), member 2; SERPINE1, serpin peptidase inhibitor, clade E, member 1; PLA2G2A, phospholipase A2, group IIA (platelets, synovial fluid). 
A

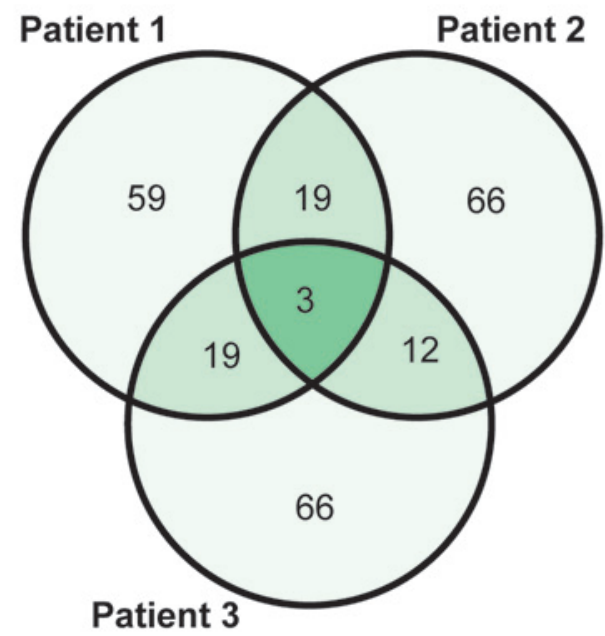

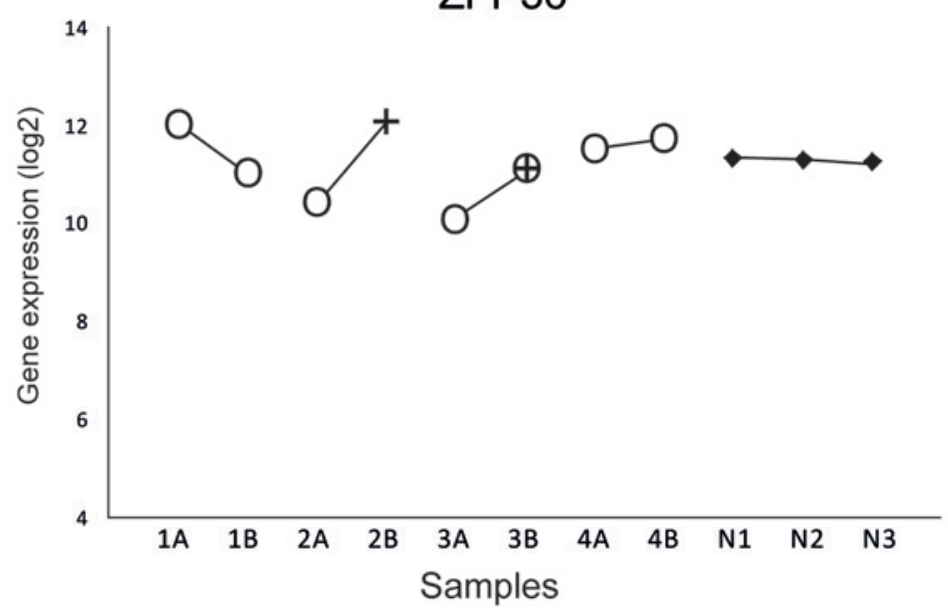

Figure 3. Chromosome 19. (A) Comparison of the most differently expressed genes from chromosome 19 within three pairs of HGSC revealed three recurrent genes: ZFP36, zinc finger protein $585 B$ and growth differentiation factor 15 . (B) ZFP36 differed $>2$-fold within all pairs of HGSC. In sample $3 B$, the comparative genomic hybridization results in cytoband $19 \mathrm{q} 13$ included both copy number gain and loss (marked with + and $\mathrm{O}$, respectively). ZFP36, zinc finger protein 36.

The present study attempted next to explore whether the copy number imbalances could partly explain the deregulation of gene expression in the selected genes of interest. For the phospholipase A2, group IIA (platelets, synovial fluid) (PLA2G2A) gene, which maps to $1 \mathrm{p} 35$, alterations at DNA and RNA level pointed in the same direction (Fig. 2D); in all HGSC pairs, one tumor had concurrent genomic loss of 1p35 and lower expression of PLA2G2A compared with the gene expression in the contralateral tumor with balanced CGH result. In the CCC pair, both tumors had copy number loss in 1p35 and PLA2G2A expression similar to the HGSC tumors with copy number loss (Fig. 2D). The normal ovarian sample used as the control exhibited PLA2G2A expression similar to that of tumor samples with balanced copy number.

However, for the majority of genes examined, no clear associations were observed between DNA imbalances and RNA expression, including the $I G J$ gene. For $I G J$ (mapping to $4 \mathrm{q} 21$ ), the gene expression varied greatly within all tumor pairs, whereas the CGH results within all tumor pairs were identical (Fig. 2A); therefore, the genomic analysis provided no indication regarding the mechanism behind the identified differences in gene expression.

Chromosome 19. The genes mapping to chromosome 19 (1,211 genes) were subjected to additional analysis. In general, the expression was more similar within the pairs for these genes than for the global gene expression data set; only $6.4 \%$ of the genes from chromosome 19 differed $>2$-fold in expression compared with $9.5 \%$ for the total set. The comparison of the most differently expressed genes within each HGSC pair revealed three recurrent genes: Zinc finger protein 36 (ZFP36, mapping on 19q13.1), zinc finger protein $585 B$ (mapping on 19q13.12) and growth differentiation factor 15 (mapping on 19p13.11) (Fig. 3A). None of these genes were differently expressed within the CCC pair. ZFP36 was also included among the most differently expressed genes for the total data set and exhibited $>2$-fold-change in expression within all three HGSC pairs (Fig. 3B).
Known ovarian cancer-related genes. In the Cancer Gene Census database, 19 genes are reported to be associated with ovarian cancer; all of which displayed similar expression within all four tumor pairs ( $<2$-fold-change difference).

\section{Discussion}

We hypothesize that bilateral ovarian carcinomas may possibly represent two primary tumors, a primary tumor and a metastasis, or two metastases. In the present study, the gene expression profiles in the bilateral carcinoma pairs from four patients were compared to assess the association between the two tumors. To the best of our knowledge, this is the first time that bilateral ovarian carcinomas have been compared in this manner. An overall similarity between the two lesions was noticed in all patients examined, thus indicating that the two tumors in each patient are clonally related. This is in agreement with former conclusions based on studies performed at the genomic level, which suggest that bilateral ovarian carcinomas have a unicentric origin $(6,7,19)$. The small number of cases examined in the present study precludes statistical testing of this conclusion, however.

The present study focused on the differently expressed genes within tumor pairs and, notably, identified among them several genes previously linked to the metastatic process in ovarian and other cancers (20-24). These results, therefore, indicate that the two tumor lesions represent different stages of tumor progression; however, since the debate on whether ovarian cancer originates from the ovarian surface epithelium or from the tube is still ongoing, it is impossible to conclude, based on the present findings, whether the ovarian lesions represent a primary tumor and its metastasis or two clonally-related metastases.

The SERPINB2 and SERPINE1 genes were among the most differentially expressed genes in all three pairs of HGSC. Both genes belong to the same gene family, encode proteins that participate in the regulation of fibrinolysis and have been linked to cancer and metastasis (23). SERPINE1 has been proposed 
to have a pro-tumorigenic function by protecting tumor cells from apoptosis, since inhibition of SERPINE1 has been demonstrated to increase spontaneous apoptosis in cancer cell lines $(25,26)$. High expression of SERPINE1 is associated with poor outcome in several cancer types, including ovarian (27), gastric (28), colorectal (29), renal (30) and breast (31) cancer. In studies of ovarian carcinomas, both SERPINB2 and SERPINE1 were upregulated in omental metastases compared with the corresponding ovarian tumors $(11,21,24)$. To the best of our knowledge, the present study demonstrates for the first time that SERPINE1 and SERPINB2 are also differently expressed within bilateral HGSC pairs.

$I G J$ was among the most differently expressed genes in all four bilateral tumor pairs (three HGSC pairs and one CCC pair). According to the Universal Protein Resource (UniProt; www.uniprot.org), the $I G J$ gene maps to $4 \mathrm{q} 21$ and encodes a protein that links two monomer units of either $\operatorname{IgM}$ or $\operatorname{IgA}$. Rearrangements involving $I G J$ have been associated with overexpression of the oncogene $v$-myc avian myelocytomatosis viral oncogene homolog in multiple myeloma, possibly leading to a more aggressive disease phenotype (32). IGJ has not previously been linked to ovarian cancer or metastasis, and its role and the mechanism behind the deregulation of gene expression require further analysis.

The gene expression data from the present study were compared with genomic aberrations. Gene expression can be altered by several mechanisms, including genomic copy number gains or losses (33). Earlier studies have reported that, in cancer, $\geq 12 \%$ of variation in gene expression is attributable to underlying variation in gene copy number $(33,34)$. The current study identified eight cytobands with different CGH results within bilateral tumor pairs, and attempted to identify an association between genetic copy number variation and expression. In the case of PLA2G2A, the tumors with loss of 1p35 (one in each HGSC pair and both CCC tumors) exhibited lower gene expression than the bilateral samples without genomic alterations. According to UniProt, this gene encodes an extracellular enzyme considered to participate in the regulation of phospholipid metabolism in biomembranes. The role of PLA2G2A in cancer is not fully understood. It has been suggested that PLA2G2A prevents carcinogenesis and metastasis by inhibiting metastasis-related genes (22). The effect seems to differ among cancer types; overexpression of PLA2G2A has been associated with both improved survival in gastric cancer (22) and poor prognosis in prostate cancer (35). Metastasis has been associated with loss of PLA2G2A expression. Ganesan et al (22) observed an inverse correlation between gene expression and cancer stage in several cancer types (gastric, colon and prostate), and suggested that genomic deletion could be one mechanism causing lower gene expression in advanced stages. To the best of our knowledge, the present study is the first to report that PLA2G2A is linked to ovarian cancer. Although cytoband 1p35 was lost in one of the bilateral HGSC in each patient, it is important to remember that the resolution of $\mathrm{CGH}$ does not provide precise information on single genes. In line with our findings, the results reported by The Cancer Genome Atlas regarding a cohort of 489 HGSC demonstrated that the genomic region harboring $P L A 2 G 2 A$ was significantly and recurrently deleted (36).
The analysis of genes mapping on chromosome 19 identified ZFP36 as differently expressed within all three HGSC pairs. According to UniProt, the protein that this gene encodes is involved in post-transcriptional gene regulation by binding adenylate-uridylate-rich elements-containing messenger RNAs and promoting their degradation. ZFP36 has been suggested to have a protective function in cancer by inhibiting metastasis-related genes, including matrix metalloproteinase (MMP)2, MMP9 and IL-6 (20,37). Loss of ZFP36 expression has been associated with metastasis (20). A study by Veskimäe et al (38) identified ZFP36 as one of the most downregulated genes when the authors compared the gene expression in BRCA1/2 germline mutated tubal and ovarian tissues (removed by risk-reducing salpingo-oophorectomy) with that in healthy control tissues, thus supporting a role for ZFP36 in ovarian carcinogenesis.

The present study has demonstrated that genes previously linked to metastasis are differently expressed within HGSC pairs in bilateral ovarian cancer. Deregulation of SERPINE1, SERPINB2, PLA2G2A and ZFP36 has previously been associated with invasion and advancing disease. However, it cannot be concluded whether bilateral tumors represent one primary tumor and a metastasis or two metastases. It would have been interesting to analyze gene expression in healthy ovarian tissue and tubal epithelium from the same patients; however, these materials were not available. The results of this study indicate that bilateral ovarian tumors represent different stages of progression during a single clonal process. However, the significance of these findings requires further analysis in larger studies.

\section{Acknowledgements}

The present study was supported by grants from the Norwegian Cancer Society (Oslo, Norway), the Inger and John Fredriksen Foundation for Ovarian Cancer Research (Oslo, Norway) and the Research Council of Norway (Oslo, Norway) through its Centers of Excellence funding scheme (project no. 179571).

\section{References}

1. Stewart B and Wild C: Cancer by organ site: Cancers of the female genital organs. World Cancer Report 2014. In: Cancer by Organ Site: Cancers of the female genital Organs. Lyon: International Agency for Research on Cancer, pp465-481, 2014.

2. Auersperg N: The origin of ovarian cancers-hypotheses and controversies. Front Biosci (Schol Ed) 5: 709-719, 2013.

3. Prat J: Ovarian carcinomas: Five distinct diseases with different origins, genetic alterations, and clinicopathological features. Virchows Arch 460: 237-249, 2012.

4. Kurman RJ, Hendrick Ellenson L and Ronnett BM (eds): Blaustein's Pathology of the Female Genital Tract. Springer, New York, NY, 2011.

5. Kurman RJ and Shih IeM: The origin and pathogenesis of epithelial ovarian cancer: A proposed unifying theory. Am J Surg Pathol 34: 433-443, 2010.

6. Pejovic T, Heim S, Mandahl N, Elmfors B, Furgyik S, Flodérus UM, Helm G, Willén H and Mitelman F: Bilateral ovarian carcinoma: Cytogenetic evidence of unicentric origin. Int J Cancer 47: 358-361, 1991.

7. Micci F, Haugom L, Ahlquist T, Abeler VM, Trope CG, Lothe RA and Heim S: Tumor spreading to the contralateral ovary in bilateral ovarian carcinoma is a late event in clonal evolution. J Oncol 2010: 646340, 2010. 
8. Khalique L, Ayhan A, Whittaker JC, Singh N, Jacobs IJ, Gayther SA and Ramus SJ: The clonal evolution of metastases from primary serous epithelial ovarian cancers. Int J Cancer 124: 1579-1586, 2009.

9. Adib TR, Henderson S, Perrett C, Hewitt D, Bourmpoulia D, Ledermann J and Boshoff C: Predicting biomarkers for ovarian cancer using gene-expression microarrays. Br J Cancer 90: 686-692, 2004

10. Lancaster JM, Dressman HK, Clarke JP, Sayer RA, Martino MA, Cragun JM, Henriott AH, Gray J, Sutphen R, Elahi A, et al: Identification of genes associated with ovarian cancer metastasis using microarray expression analysis. Int J Gynecol Cancer 16: 1733-1745, 2006

11. Brodsky AS, Fischer A, Miller DH, Vang S, MacLaughlan S, Wu HT, Yu J, Steinhoff M, Collins C, Smith PJ, et al: Expression profiling of primary and metastatic ovarian tumors reveals differences indicative of aggressive disease. PloS One 9: e94476, 2014.

12. Pejovic T, Heim S, Mandahl N, Baldetorp B, Elmfors B, Flodérus UM, Furgyik S, Helm G, Himmelmann A, Willén $\mathrm{H}$, et al: Chromosome aberrations in 35 primary ovarian carcinomas. Genes Chromosomes Cancer 4: 58-68, 1992.

13. Pejovic T, Heim S, Mandahl N, Elmfors B, Flodérus UM, Furgyik S, Helm G, Willén H and Mitelman F: Consistent occurrence of a 19p+ marker chromosome and loss of $11 p$ material in ovarian seropapillary cystadenocarcinomas. Genes Chromosomes Cancer 1: 167-171, 1989.

14. Jenkins RB, Bartelt D Jr, Stalboerger P, Persons D, Dahl RJ, Podratz K, Keeney G and Hartmann L: Cytogenetic studies of epithelial ovarian carcinoma. Cancer Genet Cytogenet 71: 76-86, 1993.

15. Taetle R, Aickin M, Yang JM, Panda L, Emerson J, Roe D, Adair L, Thompson F, Liu Y, Wisner L, et al: Chromosome abnormalities in ovarian adenocarcinoma: I. Nonrandom chromosome abnormalities from 244 cases. Genes Chromosomes Cancer 25: 290-300, 1999.

16. Smebye ML, Sveen A, Haugom L, Davidson B, Tropé CG, Lothe RA, Heim S, Skotheim RI and Micci F: Chromosome 19 rearrangements in ovarian carcinomas: Zinc finger genes are particularly targeted. Genes Chromosomes Cancer 53: 558-567, 2014.

17. Irizarry RA, Hobbs B, Collin F, Beazer-Barclay YD, Antonellis KJ, Scherf U and Speed TP: Exploration, normalization and summaries of high density oligonucleotide array probe level data. Biostatistics 4: 249-264, 2003.

18. Dysvik B and Jonassen I: J-Express: Exploring gene expression data using Java. Bioinformatics 17: 369-370, 2001

19. Bashashati A, Ha G, Tone A, Ding J, Prentice LM, Roth A, Rosner J, Shumansky K, Kalloger S, Senz J, et al: Distinct evolutionary trajectories of primary high-grade serous ovarian cancers revealed through spatial mutational profiling. J Pathol 231: 21-34, 2013

20. Al-Souhibani N, Al-Ahmadi W, Hesketh JE, Blackshear PJ and Khabar KS: The RNA-binding zinc-finger protein tristetraprolin regulates AU-rich mRNAs involved in breast cancer-related processes. Oncogene 29: 4205-4215, 2010.

21. Dorn J,Harbeck N, Kates R, Gkazepis A, Scorilas A, Soosaipillai A, Diamandis E, Kiechle M, Schmalfeldt B and Schmitt M: Impact of expression differences of kallikrein-related peptidases and of UPA and PAI-1 between primary tumor and omentum metastasis in advanced ovarian cancer. Ann Oncol 22: 877-883, 2011.

22. Ganesan K, Ivanova T, Wu Y, Rajasegaran V, Wu J, Lee MH, Yu K, Rha SY, Chung HC, Ylstra B, et al: Inhibition of gastric cancer invasion and metastasis by PLA2G2A, a novel beta-catenin/TCF target gene. Cancer Res 68: 4277-4286, 2008.

23. Heit C, Jackson BC, McAndrews M, Wright MW, Thompson DC, Silverman GA, Nebert DW and Vasiliou V: Update of the human and mouse SERPIN gene superfamily. Hum Genomics 7: 22, 2013.
24. Schmalfeldt B, Kuhn W, Reuning U, Pache L, Dettmar P, Schmitt M, Jänicke F, Höfler H and Graeff H: Primary tumor and metastasis in ovarian cancer differ in their content of urokinase-type plasminogen activator, its receptor and inhibitors types 1 and 2. Cancer Res 55: 3958-3963, 1995.

25. Fang H,Placencio VR and DeClerck YA: Protumorigenic activity of plasminogen activator inhibitor-1 through an antiapoptotic function. J Natl Cancer Inst 104: 1470-1484, 2012.

26. Mashiko S, Kitatani K, Toyoshima M, Ichimura A, Dan T, Usui T, Ishibashi M, Shigeta S, Nagase S, Miyata T and Yaegashi N: Inhibition of plasminogen activator inhibitor-1 is a potential therapeutic strategy in ovarian cancer. Cancer Biol Ther 16: 253-260, 2015

27. Kuhn W, Schmalfeldt B, Reuning U, Pache L, Berger U, Ulm K, Harbeck N, Späthe K, Dettmar P, Höfler H, et al: Prognostic significance of urokinase (uPA) and its inhibitor PAI-1 for survival in advanced ovarian carcinoma stage FIGO IIIc. Br J Cancer 79: 1746-1751, 1999.

28. Sakakibara T, Hibi K, Koike M, Fujiwara M, Kodera Y, Ito K and Nakao A: Plasminogen activator inhibitor-1 as a potential marker for the malignancy of gastric cancer. Cancer Sci 97: 395-399, 2006.

29. Berger DH: Plasmin/plasminogen system in colorectal cancer. World J Surg 26: 767-771, 2002.

30. Zubac DP, Wentzel-Larsen T, Seidal T and Bostad L: Type 1 plasminogen activator inhibitor (PAI-1) in clear cell renal cell carcinoma (CCRCC) and its impact on angiogenesis, progression and patient survival after radical nephrectomy. BMC Urol 10: 20,2010.

31. Offersen BV, Alsner J, Ege Olsen K, Riisbro R, Brünner N, Sørensen FB, Sørensen BS, Schlemmer BO and Overgaard J: A comparison among HER2, TP53, PAI-1, angiogenesis and proliferation activity as prognostic variables in tumours from 408 patients diagnosed with early breast cancer. Acta Oncol 47: 618-632, 2008

32. Affer M, Chesi M, Chen WD, Keats JJ, Demchenko YN, Tamizhmani K, Garbitt VM, Riggs DL, Brents LA, et al: Promiscuous MYC locus rearrangements hijack enhancers but mostly super-enhancers to dysregulate MYC expression in multiple myeloma. Leukemia 28: 1725-1735, 2014.

33. Pollack JR, Sørlie T, Perou CM, Rees CA, Jeffrey SS, Lonning PE, Tibshirani R, Botstein D, Børresen-Dale AL and Brown PO: Microarray analysis reveals a major direct role of DNA copy number alteration in the transcriptional program of human breast tumors. Proc Natl Acad Sci USA 99: 12963-12968, 2002.

34. Hyman E, Kauraniemi P, Hautaniemi S, Wolf M, Mousses S, Rozenblum E, Ringnér M,Sauter G,Monni O,Elkahloun A, etal: Impact of DNA amplification on gene expression patterns in breast cancer. Cancer Res 62: 6240-6245, 2002

35. Graff JR, Konicek BW, Deddens JA, Chedid M, Hurst BM, Colligan B, Neubauer BL, Carter HW and Carter JH: Expression of group IIa secretory phospholipase A2 increases with prostate tumor grade. Clin Cancer Res 7: 3857-3861, 2001.

36. Cancer Genome Atlas Research Network: Integrated genomic analyses of ovarian carcinoma. Nature 474: 609-615, 2011.

37. Van Tubergen EA, Banerjee R, Liu M, Vander Broek R, Light E, Kuo S, Feinberg SE, Willis AL, Wolf G, Carey T, et al: Inactivation or loss of TTP promotes invasion in head and neck cancer via transcript stabilization and secretion of MMP9, MMP2 and IL-6. Clin Cancer Res 19: 1169-1179, 2013.

38. Veskimäe K, Staff S, Tabaro F, Nykter M, Isola J and Mäenpää J: Microarray analysis of differentially expressed genes in ovarian and fallopian tube epithelium from risk-reducing salpingo-oophorectomies. Genes Chromosomes Cancer 54: 276-287, 2015. 